\title{
Meta-Fibonacci Sequences, Binary Trees and Extremal Compact Codes
}

\author{
Brad Jackson \\ Dept. of Mathematics \\ San Jose State University, USA \\ jackson@math.sjsu.edu
}

\author{
Frank Ruskey* \\ Dept. of Computer Science \\ University of Victoria, CANADA \\ http://www.cs.uvic.ca/〜ruskey
}

Submitted: Jun 14, 2005; Accepted: Mar 13, 2006; Published: Mar 21, 2006

Mathematics Subject Classifications: 05A15, 05A19, 68P30

\begin{abstract}
We consider a family of meta-Fibonacci sequences which arise in studying the number of leaves at the largest level in certain infinite sequences of binary trees, restricted compositions of an integer, and binary compact codes. For this family of meta-Fibonacci sequences and two families of related sequences we derive ordinary generating functions and recurrence relations. Included in these families of sequences are several well-known sequences in the Online Encyclopedia of Integer Sequences (OEIS).
\end{abstract}

\section{Introduction}

In a remarkable paper Emily Norwood studied the number of "compact codes" [7]. A compact code can be thought of as the sorted sequence of level numbers of the leaves of an extended binary tree. She provided a recurrence relation and table of the number of trees classified according to their height and their number of leaves. We will prove that if the outline of this table is considered as an increasing sequence of integers, then one of the "meta-fibonacci" numbers arises, namely the one that satisfies the recurrence relation

$$
a(n)=a(x(n)-a(n-1))+a(y(n)-a(n-2)),
$$

with $x(n)=n-1$ and $y(n)=n-2$. Sequences satisfying this recurrence, but with different linear functions for $x(n)$ and $y(n)$ have been investigated by several authors in recent years, but the general behavior of these sequences remains rather mysterious (e.g., Guy [4][Problem E31], Pinn [9]). Perhaps the most well-behaved sequences in the family

\footnotetext{
${ }^{*}$ Research supported in part by NSERC.
} 


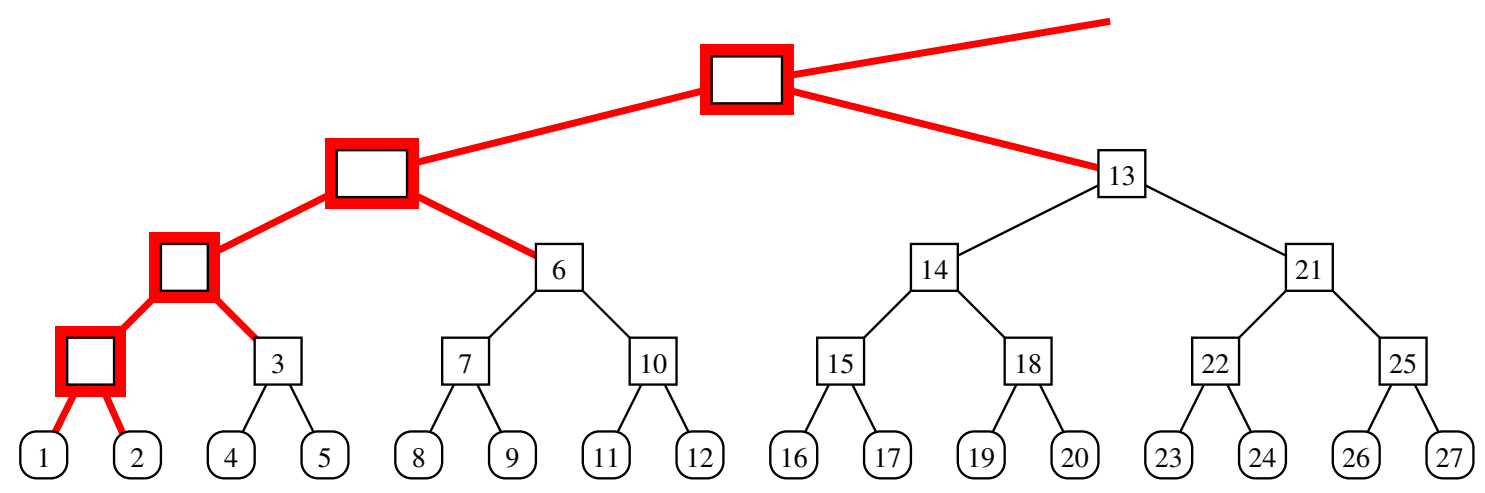

Figure 1: The tree $\mathcal{F}_{0}$.

occur when $x(n)=n$ and $y(n)=n-1$. For a given parameter $s \geq 0$, we will show that the sequences with $x(n)=y(n)+1=n-s$ are almost as well-behaved. In particular, we will show that they occur in a natural combinatorial setting, that they satisfy a recurrence relation of the form $a_{s}(n)=f(n)+a_{s}(n-g(n))$, and that they have a fairly simple ordinary generating function.

The case of $s=1$ was studied before by Tanny [10]. The case of $s=0$ was considered before by Conolly [2]. Our attempt here is to simplify, unify, generalize, and combinatorialize their results. In particular, for any fixed $s \geq 0$, we give a new way of interpreting the sequences; our interpretation is based on certain subtrees of a labeled infinite binary tree.

\section{Meta-Fibonacci Sequences and Complete Binary Trees}

Figure 1 shows part of an infinite ordered binary tree $\mathcal{F}$. The forest of labelled trees in $\mathcal{F}$ consists of a succession of complete binary trees of sizes $1,1,3,7, \ldots, 2^{h}-1, \ldots$ We refer to the subtree with $2^{h}-1$ nodes as subtree $h$, except for the leftmost subtree, which is subtree 0 . The nodes of these subtrees are labelled in preorder. Now to obtain $\mathcal{F}$ adjoin an infinite path that connects the subtrees from left-to-right as shown in Figures 1, 2 and 3 using square nodes and thickly drawn edges. We will think of this path as being parameterized by a value $s$ that gives the delay between the preorder counts of successive trees. Alternatively, we can think of the nodes along the path as being super-nodes, where each super-node contains $s$ ordinary nodes. This infinite tree is denoted $\mathcal{F}_{s}$, with our initial tree $\mathcal{F}=\mathcal{F}_{0}$. The trees $\mathcal{F}_{0}, \mathcal{F}_{1}, \mathcal{F}_{2}$ are shown in Figures $1,2,3$, respectively.

Denote by $\mathcal{T}_{s}(n)$ the tree induced by the first $n$ labelled nodes of the infinite tree $\mathcal{F}_{s}$. Define $a_{s}(n)$ to be the number of nodes at the bottom level in $\mathcal{T}_{s}(n)$. Also define $d_{s}(n)$ to be 1 if the $n$-th node is a leaf and to be 0 if the $n$-th node is an internal node. Finally, define $p_{s}(n)$ to be the positions occupied by the 1 's in the $d_{s}$ sequence. Table 1 


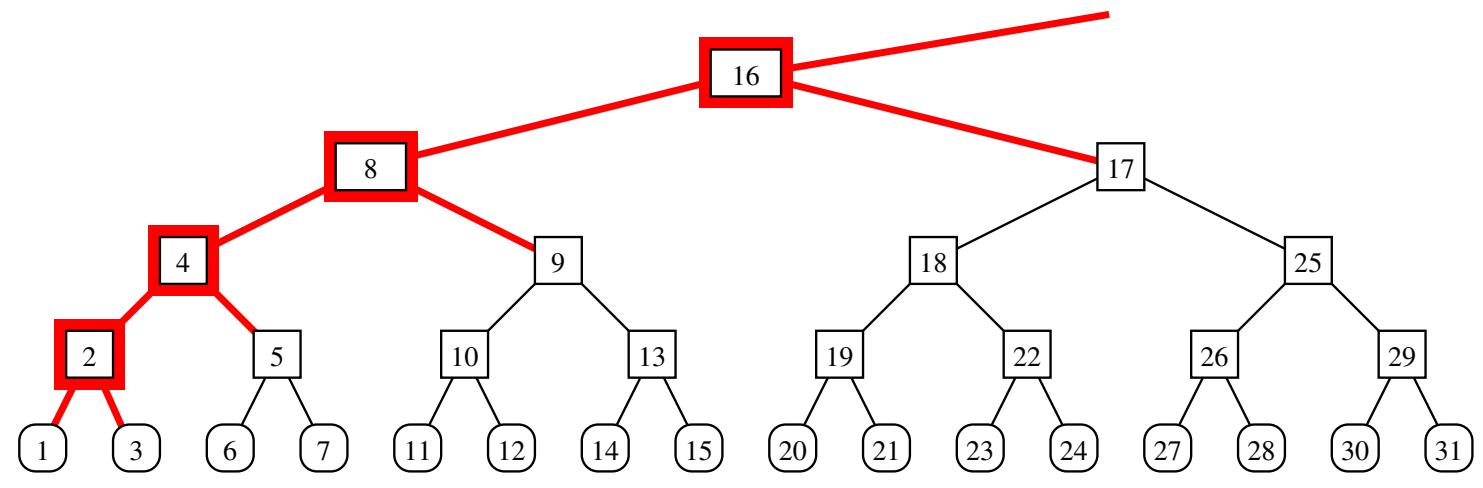

Figure 2: The tree $\mathcal{F}_{1}$.

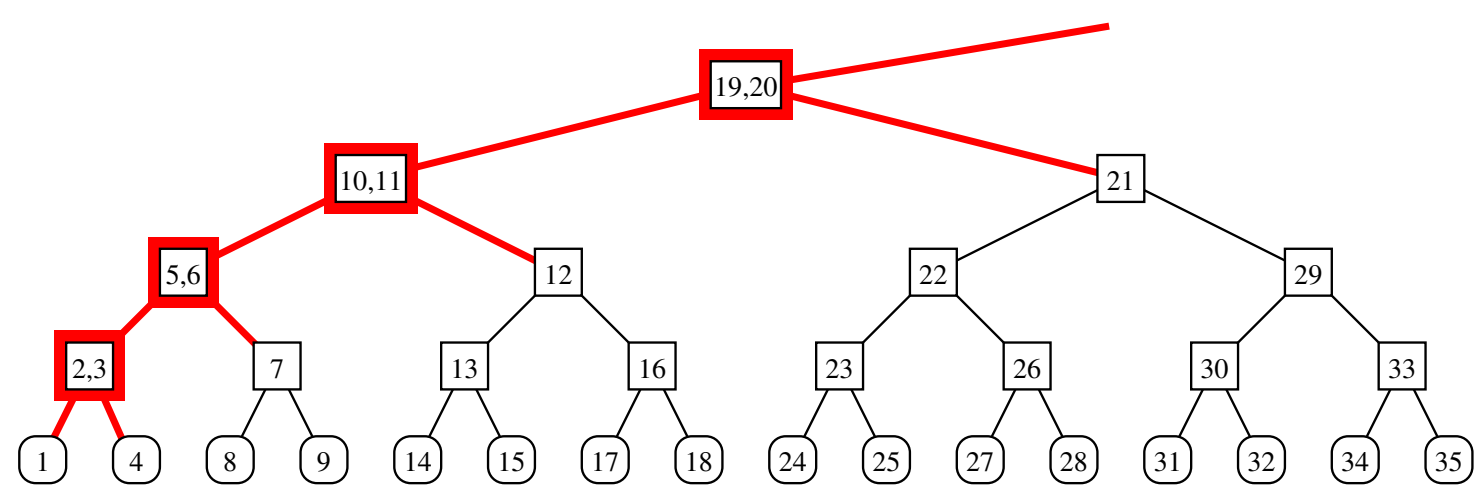

Figure 3: The tree $\mathcal{F}_{2}$.

\begin{tabular}{ccccccccccccccccccccc}
$n$ & 1 & 2 & 3 & 4 & 5 & 6 & 7 & 8 & 9 & 10 & 11 & 12 & 13 & 14 & 15 & 16 & 17 & 18 & 19 & 20 \\
\hline$a_{0}$ & 1 & 2 & 2 & 3 & 4 & 4 & 4 & 5 & 6 & 6 & 7 & 8 & 8 & 8 & 8 & 9 & 10 & 10 & 11 & 12 \\
$a_{1}$ & 1 & 1 & 2 & 2 & 2 & 3 & 4 & 4 & 4 & 4 & 5 & 6 & 6 & 7 & 8 & 8 & 8 & 8 & 8 & 9 \\
$a_{2}$ & 1 & 1 & 1 & 2 & 2 & 2 & 2 & 3 & 4 & 4 & 4 & 4 & 4 & 5 & 6 & 6 & 7 & 8 & 8 & 8 \\
\hline$d_{0}$ & 1 & 1 & 0 & 1 & 1 & 0 & 0 & 1 & 1 & 0 & 1 & 1 & 0 & 0 & 0 & 1 & 1 & 0 & 1 & 1 \\
$d_{1}$ & 1 & 0 & 1 & 0 & 0 & 1 & 1 & 0 & 0 & 0 & 1 & 1 & 0 & 1 & 1 & 0 & 0 & 0 & 0 & 1 \\
$d_{2}$ & 1 & 0 & 0 & 1 & 0 & 0 & 0 & 1 & 1 & 0 & 0 & 0 & 0 & 1 & 1 & 0 & 1 & 1 & 0 & 0 \\
\hline$p_{0}$ & 1 & 2 & 4 & 5 & 8 & 9 & 11 & 12 & 16 & 17 & 19 & 20 & 23 & 24 & 26 & 27 & 32 & 33 & 35 & 36 \\
$p_{1}$ & 1 & 3 & 6 & 7 & 11 & 12 & 14 & 15 & 20 & 21 & 23 & 24 & 27 & 28 & 30 & 31 & 37 & 38 & 40 & 41 \\
$p_{2}$ & 1 & 4 & 8 & 9 & 14 & 15 & 17 & 18 & 24 & 25 & 27 & 28 & 31 & 32 & 34 & 35 & 42 & 43 & 45 & 46
\end{tabular}

Table 1: The values of $a_{s}(n)$ and $d_{s}(n)$ for $s=0,1,2$ and $1 \leq n \leq 20$. 
gives the values of $a_{s}(n), d_{s}(n)$, and $p_{s}(n)$ for $s=0,1,2$ and $1 \leq n \leq 20$. The values of four of these table entries appear in OEIS ${ }^{1}$, namely $a_{0}(n)=A 046699, a_{1}(n)=A 006949$, $d_{0}(n)=A 079559$, and $p_{0}(n)=A 101925=A 005187(n)+1$. For fixed $s$ these numbers are related as follows.

$$
a_{s}(n)=\sum_{j=0}^{n} d_{s}(j) \quad \text { and } \quad p_{s}(n)=\min \left\{j: a_{s}(j)=n\right\} .
$$

Once we prove Theorem 2.1 below, certain results from [10] become obvious; e.g., that the numbers $a_{1}(n)$ are increasing and that their successive differences are either 0 or 1 .

The $a_{s}(n)$ numbers satisfy the meta-Fibonacci recurrence relation stated in Theorem 2.1 below.

Theorem 2.1. If $0 \leq n \leq s+1$, then $a_{s}(n)=1$. If $n=s+2$ then $a_{s}(n)=2$. If $n>s+2$, then $a_{s}(n)=a_{s}\left(n-s-a_{s}(n-1)\right)+a_{s}\left(n-s-1-a_{s}(n-2)\right)$.

Proof. First observe that if all the leaves at the last level are removed from $\mathcal{F}_{s}$, then the same structure remains, except that the leftmost super-node needs to be made into an ordinary node (by subtracting $s-1$ ). We will refer to this process as chopping the last level (also used in [7] and [3]).

We split the proof into two broad cases depending on whether $n$ is a leaf or not; i.e., whether $d_{s}(n)=1$ (Case 1) or $d_{s}(n)=0$ (Case 2).

Case 1a: If $d_{s}(n-1)=d_{s}(n)=1$ then $n$ and $n-1$ are sibling leaves and $a_{s}(n)$ is even. For example, node 28 in Figure 3. The trees $\mathcal{T}_{s}(n-1)$ and $\mathcal{T}_{s}(n-2)$ have the same number of nodes, $a_{s}(n) / 2$, at the penultimate level as does $\mathcal{T}_{s}(n)$. Thus by chopping the last level from $\mathcal{T}_{s}(n-1)$ and $\mathcal{T}_{s}(n-2)$, we see that $a_{s}\left(n-s-a_{s}(n-1)\right)=a_{s}(n) / 2=$ $a_{s}\left(n-s-1-a_{s}(n-1)\right)$.

Case 1b: If $d_{s}(n)=1$ and $d_{s}(n-1)=0$ then $n$ is a left child of its parent $n-1$ and $a_{s}(n)$ is odd. For example, node 27 in Figure 3. The tree $\mathcal{T}_{s}(n-1)$ has $\left(a_{s}(n)+1\right) / 2$ nodes at the penultimate level and the tree $\mathcal{T}_{s}(n-2)$ has $\left(a_{s}(n)-1\right) / 2$ nodes at the penultimate level. Thus by chopping the last level from $\mathcal{T}_{s}(n-1)$ and $\mathcal{T}_{s}(n-2)$, we see that $a_{s}\left(n-s-a_{s}(n-1)\right)=\left(a_{s}(n)+1\right) / 2$ and $a_{s}\left(n-s-1-a_{s}(n-1)\right)=\left(a_{s}(n)-1\right) / 2$.

Case 2a: If $d_{s}(n)=0$ and $d_{s}(n-1)=1$, then $a_{s}(n)$ is even. For example, node 26 or node 29 in Figure 3. The trees $\mathcal{T}_{s}(n-1)$ and $\mathcal{T}_{s}(n-2)$ have the same number of nodes, $a_{s}(n) / 2$, at the penultimate level. Node $n$ may have been at the penultimate level in $\mathcal{T}_{s}(n)$, but it is removed in $\mathcal{T}_{s}(n-1)$ and $\mathcal{T}_{s}(n-2)$. Thus by chopping the last level from $\mathcal{T}_{s}(n-1)$ and $\mathcal{T}_{s}(n-2)$, we see that $a_{s}\left(n-s-a_{s}(n-1)\right)=a_{s}(n) / 2=a_{s}\left(n-s-1-a_{s}(n-1)\right)$.

Case 2b: If $d_{s}(n)=0$ and $d_{s}(n-1)=0$, then $a_{s}(n)$ is even. For example, node 22 or node 30 in Figure 3. The trees $\mathcal{T}_{s}(n-1)$ and $\mathcal{T}_{s}(n-2)$ have the same number of nodes, $a_{s}(n) / 2$, at the penultimate level. Node $n$ may have been at the penultimate level in $\mathcal{T}_{s}(n)$, but it is removed in $\mathcal{T}_{s}(n-1)$ and $\mathcal{T}_{s}(n-2)$. Thus by chopping the last level from $\mathcal{T}_{s}(n-1)$ and $\mathcal{T}_{s}(n-2)$, we see that $a_{s}\left(n-s-a_{s}(n-1)\right)=a_{s}(n) / 2=a_{s}\left(n-s-1-a_{s}(n-1)\right)$.

\footnotetext{
${ }^{1}$ OEIS $=$ Neil Sloane's online encyclopedia of integer sequences.
} 
Define $\mathcal{D}_{s}$ to be the infinite string $d_{s}(1) d_{s}(2) d_{s}(3) \cdots$. Let $D_{n}$ be the finite string defined by $D_{0}=1$ and $D_{n+1}=0 D_{n} D_{n}$. Let $E_{n}$ be the finite string defined by $E_{0}=1$ and $E_{n+1}=E_{n} E_{n} 0$.

\section{Lemma 2.2.}

$$
\mathcal{D}_{0}=D_{0} D_{0} D_{1} D_{2} D_{3} \cdots=E_{\infty}
$$

Proof. The first equality in (2) is implied immediately by the definition of $\mathcal{F}_{0}$; i.e., in $0 D_{n} D_{n}$ the 0 is from the root (which is listed first in preorder) and $D_{n} D_{n}$ is from the left and right subtrees. By the definitions, $E_{n}^{R}=D_{n}$, where the superscript $R$ denotes reversal of the string. Thus

$$
D_{0} D_{0} D_{1} \cdots D_{n}=E_{n} \cdots E_{1} E_{0} E_{0} .
$$

Since $E_{n}$ is a prefix of $E_{n+1}$ by definition, the expression $E_{\infty}$ is well-defined. Hence $\mathcal{D}_{0}=E_{\infty}$.

The sequence $E_{n}$ has been considered before by Allouche, Betrema, and Shallit [1] in a different context. It is interesting to note that the sequence $\mathcal{D}_{0}$ is the limit of the morphism $0 \mapsto 0$ and $1 \mapsto 110$ (also discussed in [1], pg. 237). The following corollary is equation (6; pg. 132) in [2].

Corollary 2.3. The numbers $a_{0}(n)$ satisfy the recurrence $a_{0}\left(2^{h}-1+k\right)=2^{h-1}+a_{0}(k)$ for $0 \leq k<2^{h}$.

Proof. Since $\mathcal{D}_{0}=E_{h-1} E_{h-1} 0 \cdots$ and $\left|E_{h-1}\right|=2^{h}-1$, the value of $d_{0}\left(2^{h}-1+k\right)=d_{0}(k)$ for $1 \leq k \leq 2^{h}-1$. Since we defined $d_{0}(0)=0$ it also holds when $k=0$. The number of 1 's in $E_{h-1}$ is $\#_{1}\left(E_{h-1}\right)=2^{h-1}$. Thus

$$
\begin{aligned}
a_{0}\left(2^{h}-1+k\right) & =\sum_{j=0}^{2^{h}-1} d_{0}(j)+\sum_{j=0}^{k} d_{0}\left(2^{h}-1+j\right) \\
& =\#_{1}\left(E_{h-1}\right)+\sum_{j=0}^{k} d_{0}(j) \\
& =2^{h-1}+a_{0}(k) .
\end{aligned}
$$

\section{Lemma 2.4.}

$$
a_{s}(n)= \begin{cases}a_{0}(n-s h) & \text { if } 2^{h}+(s-1) h+1 \leq n \leq 2^{h+1}+(s-1) h-1, \\ 2^{h-1} & \text { if } 2^{h}+(s-1) h-s+1 \leq n \leq 2^{h}+(s-1) h .\end{cases}
$$


Proof. The labels on the nodes in subtree $h$ in $\mathcal{F}_{s}$ are exactly the values of $n$ lying in the first range above. This is true since there are $1+1+3+\cdots+\left(2^{h-1}-1\right)=2^{h}-h$ nodes in the subtrees to the left of subtree $h$, and $s h$ super-nodes. Thus the lowest label of a node in subtree $h$ is $2^{h}-h+s h+1=2^{h}+(s-1) h+1$, and the highest label is $2^{h}+(s-1) h+2^{h}-1$. The difference between the labels on corresponding nodes in $\mathcal{F}_{s}$ and $\mathcal{F}_{0}$ is $s h$ if the nodes are in subtree $h$; thus $a_{s}(n)=a_{0}(n-s h)$.

In the second range the nodes are super-nodes lying between subtrees $h-1$ and $h$ and therefore having $2^{h-1}$ leaves in their left-subtree.

\section{Corollary 2.5.}

$$
a_{1}(n)=a_{0}(n-\lfloor\lg n\rfloor) .
$$

Proof. Taking $s=1$ in Lemma 2.4 we obtain $a_{1}(n)=a_{0}(n-h)$ in the range $2^{h}+1 \leq$ $n \leq 2^{h+1}-1$. In that range $h=\lfloor\lg n\rfloor$. We need only check what happens when $n=2^{h}$. By the lemma $a_{1}\left(2^{h}\right)=2^{h-1}$. However, in $\mathcal{F}_{0}$ the node $2^{h}-h$ is the rightmost node in subtree $h$ and thus $a_{0}\left(2^{h}-h\right)=2^{h-1}$.

The case $s=1$ of the theorem below is roughly equivalent to equations (2.2) and (2.3) in Tanny [10]. For proposition $P$ the notation $\llbracket P \rrbracket$ means 1 if $P$ is true and 0 if $P$ is false.

Theorem 2.6. If $1 \leq k \leq 2^{h-1}-1$, then

$$
a_{s}\left(2^{h}+(s-1) h+k+1\right)=2^{h-2}+a_{s}\left(2^{h-1}+(s-1) h-s+k+1\right) .
$$

If $1 \leq k \leq 2^{h-1}-1$, then

$$
a_{s}\left(2^{h}+2^{h-1}+(s-1) h+k\right)=2^{h-1}+a_{s}\left(2^{h-1}+(s-1) h-s+k+1\right) .
$$

If $2^{h}+(s-1) h-s+1 \leq n \leq 2^{h}+(s-1) h+1$, then $a_{s}(n)=2^{h-1}+\llbracket n=s+2 \rrbracket$.

Proof. Let the node $n$ be in the subtree $h$ or the super-node, call it $y$, that is the parent of subtree $h$. Let $x$ be the root of that subtree and denote the left and right subtrees of $x$ by $T_{L}$ and $T_{R}$. We will prove the following recurrence relation.

$$
a_{s}(n)= \begin{cases}2^{h-1}+a_{s}\left(n-2^{h}-s+1\right) & \text { if } n \in T_{R}, \\ 2^{h-2}+a_{s}\left(n-2^{h-1}-s\right) & \text { if } n \in T_{L}, \\ 2^{h-1}+\llbracket n=s+2 \rrbracket & \text { if } n=x \text { or } n \in y .\end{cases}
$$

Let $T$ be the subtree whose root is the right child of the left child of $y$. In the first two cases above we are mappping the subtree $T_{L}$ or $T_{R}$ to $T$, which has the same structure. In the case of $T_{R}$ we skip over $2^{h-1}$ leaves and $2^{h}+s-1$ nodes. In the case of $T_{L}$ we skip over $2^{h-2}$ leaves and $2^{h-1}+s$ nodes. In the remaining case, if $n=x$ or $n \in y$, then $a_{s}(n)=2^{h-1}+\llbracket n=s+2 \rrbracket$; the test for $n=s+2$ is necessary because subtree 0 is special.

From the proof of the previous lemma we know that $x=2^{h}+(s-1) h+1$ and thus that the root of $T_{R}$ is $2^{h}+2^{h-1}+(s-1) h+1$ and the root of $T_{L}$ is $x+1=2^{h}+(s-1) h+2$. Thus we know the exact range of $n$ in each of the subtrees and the theorem statement is another way of writing (3). 
Let $r_{1}, r_{2}, r_{3}, r_{4}, \ldots=1,2,1,3,1,2,1,4,1,2,1,3,1,2,1 \ldots$ be the transition sequence of the binary reflected Gray code; this sequence is also known as the "ruler function" (A001511). If the alternating 0's are removed from the sequence $r_{1}-1, r_{2}-1, r_{3}-1, r_{4}-$ $1, \ldots=0,1,0,2,0,1,0,3,0,1,0,2,0,1,0 \ldots$ then the ruler function is again obtained. This implies that the generating function, $R(z)$, of the ruler function satisfies the functional equation $R(z)=z /(1-z)+R\left(z^{2}\right)$. This equation can be iterated to obtain

$$
R(z)=\sum_{k \geq 1} r_{k} z^{k}=\sum_{n \geq 0} \frac{z^{2^{n}}}{1-z^{2^{n}}} .
$$

\section{Lemma 2.7.}

$$
\begin{aligned}
\mathcal{D}_{0} & =110^{r_{1}} 110^{r_{2}} 110^{r_{3}} 110^{r_{4}} \ldots \\
& =10^{r_{1}-1} 10^{r_{2}-1} 10^{r_{3}-1} 10^{r_{4}-1} \ldots
\end{aligned}
$$

Proof. The ruler sequence is $R_{\infty}$ where $R_{1}=1$ and $R_{n+1}=R_{n}, n+1, R_{n}$. Since $\left|R_{n}\right|=$ $2^{n}-1$, we have $r_{2^{n}+i}=r_{i}$ for $1 \leq i \leq 2^{n}-1$ and $r_{2^{n}}=n+1$. We will show that

$$
E_{n}=110^{r_{1}} 110^{r_{2}} \cdots 110^{r_{2}-1},
$$

which will finish the proof of the first equality since $\mathcal{D}_{0}=E_{\infty}$. By induction

$$
\begin{aligned}
E_{n+1} & =E_{n} E_{n} 0 \\
& =110^{r_{1}} 110^{r_{2}} \cdots 110^{r_{2} 2^{n-1}} 110^{r_{1}} 110^{r_{2}} \cdots 110^{r_{2^{n-1}}} 0 \\
& =110^{r_{1}} 110^{r_{2}} \cdots 110^{r_{2^{n-1}}} 110^{r_{1+2^{n-1}}} 110^{r_{2+2^{n-1}}} \cdots 110^{r_{2^{n}-1}} 110^{n+1},
\end{aligned}
$$

as required. The second equality follows from the well-known property of the ruler sequence that $R_{\infty}=1+\left(0, r_{1}, 0, r_{2}, 0, r_{3}, 0, r_{4}, 0, \ldots\right)$.

We can extend some of the previous results about $\mathcal{D}_{0}$ to $\mathcal{D}_{s}$.

Lemma 2.8. Let $s_{j}=r_{j}+s \llbracket j$ is a power of $2 \rrbracket$.

$$
\begin{aligned}
& \mathcal{D}_{s}=D_{0} 0^{s} D_{0} 0^{s} D_{1} 0^{s} D_{2} 0^{s} D_{3} 0^{s} \ldots \\
& \mathcal{D}_{s}=10^{s_{1}-1} 10^{s_{2}-1} 10^{s_{3}-1} 10^{s_{4}-1} \ldots
\end{aligned}
$$

Proof. The proof is similar to those used in Lemmata 2.7 and 2.2 and is omitted.

Since the $p_{s}(n)$ numbers give the positions of the 1 's in $\mathcal{D}_{s}$ the following corollary is true.

Corollary 2.9. For all $n \geq 1$,

$$
p_{s}(n+1)-p_{s}(n)=r_{n}+s \llbracket n \text { is a power of } 2 \rrbracket \text {. }
$$




\subsection{Generating Functions}

If $S=s(1) s(2) \cdots s(m)$ is a string then we use $S(z)$ to denote the ordinary generating function $S(z)=\sum s(i) z^{i}$. Let $\mathcal{A}_{s}(z)$ and $\mathcal{D}_{s}(z)$ denote the ordinary generating functions of the $a_{s}(n)$ and $d_{s}(n)$ sequences, respectively. Directly from the definitions we get the equation shown below:

$$
\mathcal{A}_{s}(z)=\frac{\mathcal{D}_{s}(z)}{1-z} .
$$

Since $\mathcal{A}_{s}(z)$ is determined by $\mathcal{D}_{s}(z)$ and $\mathcal{D}_{s}(z)$ is easier to treat, we first concentrate our attention on $\mathcal{D}_{s}(z)$.

Lemma 2.10.

$$
\begin{aligned}
& D_{n}(z)=z^{n+1}(1+z)\left(1+z^{3}\right) \cdots\left(1+z^{2^{n}-1}\right)=z^{n+1} \prod_{j=1}^{n}\left(1+z^{2^{j}-1}\right) . \\
& E_{n}(z)=z(1+z)\left(1+z^{3}\right) \cdots\left(1+z^{2^{n}-1}\right)=z \prod_{j=1}^{n}\left(1+z^{2^{j}-1}\right) .
\end{aligned}
$$

Proof. From the recurrence relation $D_{0}=1$ and $D_{n+1}=0 D_{n} D_{n}$ we obtain $D_{0}(z)=z$ and

$$
D_{n+1}(z)=z D_{n}(z)+z^{\left|0 D_{n}\right|} D_{n}(z)=z\left(1+z^{2^{n+1}-1}\right) D_{n}(z) .
$$

Similarly $E_{0}(z)=z$ and $E_{n+1}(z)=\left(1+z^{2^{n+1}-1}\right) E_{n}(z)$. The result now follows by induction.

Corollary 2.11.

$$
\mathcal{D}_{0}(z)=z(1+z)\left(1+z^{3}\right)\left(1+z^{7}\right) \cdots=z \prod_{n \geq 1}\left(1+z^{2^{n}-1}\right) .
$$

Proof. Follows at once from the the preceding lemma and the equation $\mathcal{D}_{0}=E_{\infty}$ from Lemma 2.2.

Theorem 2.12. The generating function $\mathcal{D}_{s}(z)$ is equal to

$$
z\left(1+z^{s+2^{0}}\left(1+z^{s+2^{1}}\left[1+z^{2^{1}-1}\right]\left(1+z^{s+2^{2}}\left[1+z^{2^{2}-1}\right]\left(1+z^{s+2^{3}}\left[1+z^{2^{3}-1}\right](1+\cdots\right.\right.\right.\right.
$$

Proof. We need to translate the string $D_{0} 0^{s} D_{0} 0^{s} D_{1} 0^{s} D_{2} 0^{s} D_{3} 0^{s} \cdots$ from Lemma 2.8 into its generating function. Since

$$
\left|D_{0} 0^{s} D_{0} 0^{s} D_{1} 0^{s} \cdots D_{n-1} 0^{s}\right|=s+1+\sum_{j=0}^{n-1}\left(2^{j+1}-1+s\right)=2^{n+1}+(s-1)(n+1),
$$


we can write

$$
\mathcal{D}_{s}(z)=z+\sum_{n \geq 0} z^{2^{n+1}+(n+1)(s-1)} D_{n}(z)=z+\sum_{n \geq 0} z^{2^{n+1}+(n+1)(s-1)+1} x_{1} x_{2} \cdots x_{n},
$$

where $x_{k}=z\left(1+z^{2^{k}-1}\right)$, so that $D_{n}(z)=z x_{1} x_{2} \cdots x_{n}$. Now rewrite $(5)$ as

$$
z\left(1+z^{s+2^{0}}\left(1+z^{s+2^{1}-1} x_{1}\left(1+z^{s+2^{2}-1} x_{2}\left(1+z^{s+2^{3}-1} x_{3}(1+\cdots\right.\right.\right.\right.
$$

In (8), the coefficient of $x_{1} x_{2} \cdots x_{n}$ is $z$ raised to the power $1+2^{n+1}+(s-1)(n+1)$ by the sum given in (6), agreeing with (7).

Theorem 2.13. If $s \geq 1$, then

$$
\mathcal{A}_{s}(z)=\frac{1-z^{s}}{1-z}\left(z+z \sum_{n \geq 1} \prod_{k=1}^{n} z^{s-1}\left(z+z^{2^{k}}\right)\right)
$$

Proof. Call the expression on the right $R_{s}(z)$ and let $y=z^{s-1}$. Multiply $R_{s}(z)$ by $1-z$, expand, and collect terms by increasing powers of $y$ to obtain

$$
\begin{aligned}
(1-z) R_{s}(z) & =(1-z y)\left(z+z \sum_{n \geq 1} \prod_{k=1}^{n-1} y\left(z+z^{2^{k}}\right)\right) \\
& =z+z \sum_{n \geq 1} y^{n} \prod_{k=1}^{n}\left(z+z^{2^{k}}\right)-z^{2} y-z^{2} y \sum_{n \geq 1} \prod_{k=1}^{n} y^{n}\left(z+z^{2^{k}}\right) \\
& =z+z \sum_{n \geq 1} y^{n}\left(\prod_{k=1}^{n}\left(z+z^{2^{k}}\right)-z y \prod_{k=0}^{n}\left(z+z^{2^{k}}\right)\right) \\
& =z+z \sum_{n \geq 1} y^{n}\left(\left(z+z^{2^{n}}\right) \prod_{k=1}^{n-1}\left(z+z^{2^{k}}\right)-z \prod_{k=0}^{n-1}\left(z+z^{2^{k}}\right)\right) \\
& =z+z \sum_{n \geq 1} y^{n} z^{2^{n}} \prod_{k=1}^{n-1}\left(z+z^{2^{k}}\right)
\end{aligned}
$$

Note that this last expression is equal to $\mathcal{D}_{s}(z)$ by $(7)$.

Jon Perry [8] has observed experimentally that $a_{1}(n)$ counts the number of compositions of $n$ such that, for some $m$,

$$
x_{0}+x_{1}+\cdots+x_{m}=n \quad \text { where } \quad x_{i} \in\left\{1,2^{i}\right\} \text { for } i=0,1, \ldots, m .
$$

He uses the notation $1+[1,2]+[1,4]+[1,8]+\cdots$ to denote the set of such compositions and notes that many other combinatorial objects are in one-to-one correspondence with similar composition rules [8]. We call these rules specifications. 
Corollary 2.14. For $s \geq 1$, the number of compositions of $n$ with specification

$$
[1,2, \ldots, s]+[s, 2+s-1]+[s, 4+s-1]+[s, 8+s-1]+\cdots
$$

is $a_{s}(n)$. For $s=0$, the corresponding specification is

$$
[\mathbb{N}]+[0,1]+[0,3]+\cdots+\left[0,2^{j}-1\right]+\cdots
$$

Proof. This is clear from the generating function for $\mathcal{A}_{s}(z)$ given in Theorem 2.13 once $z\left(1-z^{s}\right) /(1-z)$ is written as $z+z^{2}+\cdots+z^{s}$.

As an example, for $s=2$ and $n=8$, the specification is $[1,2]+[2,3]+[2,5]+[2,9]+\cdots$ and the $a_{2}(8)=3$ compositions are

$$
8=1+2+5=1+3+2+2=2+2+2+2 .
$$

To finish this section we also develop a generating function for the $p_{s}(n)$ sequences.

Lemma 2.15. For all $s \geq 0$,

$$
\sum_{n \geq 0} p_{s}(n) z^{n}=\frac{1}{1-z}\left(1+z \sum_{k \geq 0} z^{2^{k}}\left(s+\frac{1}{1-z^{2^{k}}}\right)\right) .
$$

Proof. Let $\mathcal{P}_{s}(z)$ denote the ordinary generating function of the numbers $p_{s}(n)$. Then

$$
\sum_{n \geq 1}\left(p_{s}(n+1)-p_{s}(n)\right) z^{n}=\frac{1}{z}\left((1-z) \mathcal{P}_{s}(z)-1\right) .
$$

By Corollary 2.9 this expression is equal to

$$
\sum_{n \geq 1}\left(r_{n}+s \llbracket n \text { is a power of } 2 \rrbracket\right) z^{n}=\sum_{k \geq 0}\left(s z^{2^{k}}+\frac{z^{2^{k}}}{1-z^{2^{k}}}\right),
$$

where the equality follows from (4). Solving for $\mathcal{P}_{s}(z)$ finishes the proof.

\section{Binary Compact Codes}

A binary compact code can be represented by an extended binary tree. We use the term extended binary tree in the sense of Knuth [6]: every node has either no children (a leaf) or two children (an internal node). Since no other types of codes are considered here, we shorten "binary compact code" to "code". A code of order $n$ can be represented by a tree with $n$ leaves in which the level numbers $\ell_{1} \geq \ell_{2} \geq \cdots \geq \ell_{n}$ of the leaves are non-increasing. We will identify a compact binary code by the sequence of level numbers $\left(\ell_{1}, \ell_{2}, \ldots, \ell_{n}\right)$. For example, the codes for $n=5$ are $(3,3,3,3,1),(3,3,2,2,2)$, and $(4,4,3,2,1)$. Every code of order $n$ corresponds to a unique partition of 1 into the $n$ 
powers of $1 / 2$ given by $1=2^{-\ell_{1}}+2^{-\ell_{2}}+\cdots+2^{-\ell_{n}}$. Thus $(3,3,3,3,1)$ corresponds to the partition $1=1 / 8+1 / 8+1 / 8+1 / 8+1 / 2$.

The height $h$ of a tree is the length of the longest path from the root to any leaf. For a given height $h$ and integer $n$, we consider here the problem of finding the maximum number of leaves at the largest level $h$ among all trees with $n$ leaves, which we denote by $M(n, h)$. Clearly $M(n, h)=0$ if $h<\lceil\lg n\rceil$. A tree $T$ with $n$ vertices and height $h$ that has $M(n, h)$ leaf pairs at the largest level is said to be an optimal tree. We will show that our first two meta-Fibonacci sequences can be realized by certain families of optimal trees. This will be done via a "greedy" algorithm for constructing a sequence of optimal tree/codes for successive values of $n$ and a fixed value $h$. We denote these trees $\mathcal{T}_{n, h}$ for natural numbers $n$ and $h$ and call them greedy trees. Here is the greedy algorithm for constructing $\mathcal{T}_{n, h}$.

- If $n=h+1$, then there is only one tree/code, namely $h, h, h-1, \ldots, 2,1$.

- Given $\mathcal{T}_{n, h}$ the code $\mathcal{T}_{n, h}$ is obtained by replacing the leftmost level $\ell_{i}$ for which $\ell_{i}<h$ by the two levels $\ell_{i}+1, \ell_{i}+1$.

We will also consider the trees $\mathcal{T}_{n}=\mathcal{T}_{n,\lceil\lg n\rceil}$. They may be constructed greedily as follows.

- If $n=0$, then the tree is a leaf.

- If $n=2^{h}$, then $\mathcal{T}_{n}$ is a complete binary tree (all leaves are at level $h$ ). Tree $\mathcal{T}_{n+1}$ is the tree of height $h+1$ whose left subtree is $\mathcal{T}_{n}$ and whose right subtree is a single leaf.

- If $n$ is not a power of 2, then expand the leftmost leaf which is not at the largest level, as described above.

It is also interesting to consider the inverse process of obtaining $\mathcal{T}_{n}$ from $\mathcal{T}_{n+1}$. The inverse rule is very simple: Replace the rightmost equal pair $\ell_{j}=\ell_{j+1}$ by $\ell_{j}-1$.

We could also have defined a code by the number of internal nodes at each level in the corresponding tree. Given a code of height $h$, let $\left[\tau_{0}, \tau_{1}, \ldots, \tau_{h-1}\right]$ be the sequence in which $\tau_{i}$ is the number of internal nodes at level $i$. For our example codes given earlier, the corresponding level counts are $[1,1,2],[1,2,1],[1,1,1,1]$. These counts clearly must satisfy

$$
\begin{aligned}
& \tau_{i} \leq 2 \tau_{i-1}, \text { for } 1 \leq i \leq h-1 \text { and } \\
& \tau_{0}+\tau_{1}+\cdots+\tau_{h-1}=n-1 .
\end{aligned}
$$

Subject to these two constraints $M(n, h)$ is the largest value that $\tau_{h-1}$ can attain.

Let $k$ be the largest level for which $\tau_{k}<2 \tau_{k-1}$. The greedy algorithm simply replaces $\tau_{k}$ by $1+\tau_{k}$. 
Lemma 3.1. Let $\tau_{0}, \tau_{1}, \ldots, \tau_{h-1}$ be the number of vertices at each level for the tree $\mathcal{T}_{n, h}$ and suppose that $t_{0}, t_{1}, \ldots, t_{h-1}$ are the vertex numbers by level for any other tree $T$ with $n$ leaves and height $h$. For any $0 \leq j \leq h-1$,

$$
\tau_{j}+\cdots+\tau_{h-1} \geq t_{j}+\cdots+t_{h-1} .
$$

Proof. For any $h$, the result is true for $n=h+1$ since there is only one tree with $h+1$ leaves and height $h$. Similarly it is true for $n=2^{h}$.

Assume the result is true for all trees with $n$ (for some $n<2^{h}$ ) leaves and height $h$. Let $\tau_{1}^{\prime}, \tau_{2}^{\prime}, \ldots, \tau_{h-1}^{\prime}$ be the vertex numbers by level for $\mathcal{T}_{n+1, h}$. Note that for some $k>0$, we have $\tau_{k}^{\prime}=1+\tau_{k}$ and $\tau_{j}^{\prime}=\tau_{j}$ for all $j \neq k$. By the greedy algorithm $\tau_{h-1}=2 \tau_{h-2}, \ldots, \tau_{k+1}=2 \tau_{k}$, but $\tau_{k}<2 \tau_{k-1}$. Let $T^{\prime}$ be any tree with $n+1$ leaves and height $h$ and suppose that $T$ is the tree with $n$ leaves and height $h$ formed by removing from $T^{\prime}$ the rightmost pair of leaves at level $h$. Suppose that $t_{0}, t_{1}, \ldots, t_{h-1}$ are the vertex numbers by level for $T$. By induction, we assume that $t_{j}+\cdots+t_{h-2}+t_{h-1} \leq \tau_{j}+\cdots+\tau_{h-2}+\tau_{h-1}$ for all $0 \leq j \leq h-1$. Let $t_{0}^{\prime}, t_{1}^{\prime}, \ldots, t_{h-1}^{\prime}$ be the vertex numbers by level for for $T^{\prime}$. Note that $t_{h-1}^{\prime}=1+t_{h-1}$ and $t_{j}^{\prime}=t_{j}$ for all $j<h-1$. For all $i \leq k$, we see that $t_{i}^{\prime}+\cdots+t_{h-1}^{\prime} \leq \tau_{i}^{\prime}+\cdots+\tau_{h-1}^{\prime}$.

Suppose $t_{i}^{\prime}+\cdots+t_{h-1}^{\prime}>\tau_{i}^{\prime}+\cdots+\tau_{h-1}^{\prime}$ for some $i>k$. Let $m \geq i$ be the smallest index such that $t_{m}^{\prime}>\tau_{m}^{\prime}=\tau_{m}$. Since $m>k$ we have $\tau_{m}=2 \tau_{m-1}=2 \tau_{m-1}^{\prime}$. Since $m$ was chosen to be smallest $\tau_{m-1}^{\prime} \geq t_{m-1}^{\prime}$. Putting these inequalities together we have $t_{m}^{\prime}>2 t_{m-1}^{\prime}$ which is a contradiction. Thus $t_{i}^{\prime}+\cdots+t_{h-1}^{\prime} \leq \tau_{i}^{\prime}+\cdots+\tau_{h-1}^{\prime}$ for all $i$ and the result is true by induction.

Theorem 3.2. The greedy algorithm produces optimal trees.

Proof. Let $t_{h-1}$ be the number of internal nodes in some code with $n$ leaves and height $h$. The previous lemma tells us that $\tau_{h-1} \geq t_{h-1}$, where $\tau_{h-1}$ is the number of internal nodes at level $h-1$ in $\mathcal{T}_{n, h}$.

Corollary 3.3. The largest number of 1 's in a partition of $2^{h}$ into powers of 2 consisting of $n$ parts is $M(n, h)$.

Proof. Multiply the partition of 1 described above by $2^{h}$ to obtain a partition of $2^{h}$.

Define $a(n)$ to be the maximum number of leaf pairs at the largest level, taken over all binary trees with $n$ leaves. In other words, $a(n)=\max M(n, h): 1 \leq h \leq n-1$.

Corollary 3.4. For any $n$, we have $a(n)=M(n,\lceil\lg n\rceil)=a_{1}(n-1)$.

Proof. For any $k>h=\lceil\lg n\rceil$ construct a optimal tree of height $k$ with $n$ leaves using the greedy algorithm. The tree $\mathcal{T}_{n, k}$ has a subtree attached to an interior vertex at level $k-h$ which is ismorphic to $\mathcal{T}_{n-k+h, h}$. Clearly $M(n-k+h, h) \leq M(n, h)$ and thus $a(n)=M(n,\lceil\lg n\rceil)$. The tree $\mathcal{T}_{1}(n)$ from Section 2 which defines the sequence $a_{1}(n)$ has $n+1$ leaves (since it has $n$ interior vertices) and is equal to the greedy tree for $M(n+1,\lceil\lg (n+1)\rceil)$. Only the order in which the vertices are added is different since we add the vertices from the bottom in constructing $\mathcal{T}_{1}(n)$. 
Similarly define $b(n)$ by the equation $b(n)=M(n+h, h)$ for $h+1 \leq n+h \leq 2^{h}$.

Corollary 3.5. The sequence $b$ is well-defined and $b(n)=a_{0}(n)$.

Proof. For a given $n$, let $h$ be the smallest height such that $n+h \leq 2^{h}$. For any larger height $k>h$ construct a optimal tree of height $k$ with $n+k$ leaves using the greedy algorithm. The tree $\mathcal{T}_{n+k, k}$ has a subtree attached to an interior vertex at level $k-h$ which is equal to $\mathcal{T}_{n+h, h}$. Thus $M(n+k, k)=M(n+h, h)$. If $h$ is the height of the $n$th subtree $\mathcal{T}_{n}$ which defines the sequence $a_{0}$, we see inductively that $\mathcal{T}_{n}$ has $n+h-1$ internal nodes and thus $n+h$ leaves. As before we see that $T_{n}$ is equal to the greedy tree for $M(n+h, h)$. Thus $\mathcal{T}_{n}$ has $b(n)$ leaf pairs at the largest level $h$, and we see that $b(n)=a_{0}(n)$.

Thus we have shown that the first 2 meta-Fibonacci sequences in our family of sequences have concrete realizations as the solutions of optimization problems involving binary compact codes/trees.

Acknowledgements: We wish to thank Don Knuth, Jon Perry, Jeff Shallit, Herb Wilf, and Chris Deugau for helpful comments related to this research.

\section{References}

[1] J.-P. Allouche, J. Betrema, and J.O. Shallit, Sur des Points Fixes de Morphismes d'un Monoïde Libre, Informatique théorique et Applications, 23 (1989) 235-249.

[2] B.W. Conolly, Meta-Fibonacci Sequences, Chapter XII in S. Vajda, Fibonacci \& Lucas Numbers, and the Golden Section, Ellis Horwood Limited, 1989.

[3] P. Flajolet and H. Prodinger, Level Number Sequences for Trees, Discrete Mathematics 65 (1987) 149-156.

[4] R.K. Guy, Unsolved Problems in Number Theory, Problem Books in Math., Springer, New York, 1981.

[5] M. Khosravifard, M. Esmaeili, H. Saidi, and T.A. Gulliver, A Tree Based Algorithm for Generating All Possible Binary Compact Codes with N Codewords, IEICE Transactions on Fundamentals of Electronics, Communications and Computer Sciences, Vol. E86-A (2003) 2510-2516.

[6] D.E. Knuth, The Art of Computer Programming, Volume 1: Fundamental Algorithms, Addison-Wesley, 1968.

[7] Emily Norwood, The Number of Different Possible Compact Codes, IEEE Transactions on Information Theory, vol. IT-13, no. 4, pp. 613-616, 1967.

[8] Jon Perry, Symmetric Ferrar Diagrams, website, www.users.globalnet.co.uk/ nerry/maths/symmetricferrars/symmetricferrars.htm, March 2005.

[9] K. Pinn, Order and chaos in Hofstadter's Q(n) sequence, Complexity 4 (1999) 41-46.

[10] S.M. Tanny, A well-behaved cousin of the Hofstadter sequence, Discrete Mathematics, 105 (1992) 227-239. 


\section{Corrigendum - submitted November 15, 2007}

Here we list and fix some small errors/typos that occur in the paper:

Thanks to Steve Tanny and his students, Bala Balamohan and Li Zhiqang, for pointing these out to us. We also make some remarks about references and extensions.

- In the definition of $\mathcal{T}_{s}(n)$ on page 2 , by the "first $n$ nodes" we are referring to the labels on the nodes.

- In equation (1) the summation should start with $j=1$.

- In the proof of Theorem 2.1, at the end of each case there is an $a_{s}\left(n-s-1-a_{s}(n-1)\right)$ that occurs. In each case it should be $a_{s}\left(n-s-1-a_{s}(n-2)\right)$ instead.

- The proof of Theorem 2.13 is somewhat flawed. On the next page we provide a better statement of this theorem and its proof.

- In the proof of Lemma 2.15 there are two places where a 1 should be a $z$ : First in the statement of the Lemma the right hand side should be

$$
\frac{1}{1-z}\left(z+z \sum_{k \geq 0} z^{2^{k}} \frac{1}{1-z^{2^{k}}}\right) .
$$

Second, the right hand side of the first equation in the proof should be $\frac{1}{z}((1-$ $\left.z) \mathcal{P}_{s}(z)-z\right)$. Note that these changes are only because the constant term of $\mathcal{P}_{s}(z)$ is 0 , not 1 .

- Some of these results have been extended to $k$-ary trees in the paper C. Deugau and F. Ruskey, Complete k-ary Trees and Generalized Meta-Fibonacci Sequences, Fourth Colloquium on Mathematics and Computer Science: Algorithms, Trees, Combinatorics and Probabilities, September 18-22, 2006, Institut lie Cartan, Nancy, France, 2006. DMTCS Proceedings Series, Volume AG, 203-214.

- The website of Jon Perry seems to have disappeared (reference [8]) but a copy may be found at web.archive.org/web/20060515224323/www . users.globalnet.co.uk/ perry/ maths/symmetricferrars/symmetricferrars.htm.

- Recent papers about sequences related to those discussed here include: Callaghan, Chew, and Tanny, On the behavior of a family of meta-Fibonacci sequences, SIAM J. Discrete Math. 18 (2005) 794-824; Balamohan, Kuznetsov, and Tanny, On the Behavior of a Variant of Hofstadter's Q-Sequence, Journal of Integer Sequences, Vol. 10 (2007), Article 07.7.1. 
Theorem 2.13 (revised). If $s \geq 1$, then

$$
\mathcal{A}_{s}(z)=z \frac{1-z^{s}}{1-z} \sum_{n \geq 0} \prod_{k=1}^{n} z^{s-1}\left(z+z^{2^{k}}\right) .
$$

Proof. Call the expression on the right $R_{s}(z)$ and let $y=z^{s-1}$. Multiply $R_{s}(z)$ by $1-z$, expand, and collect terms by increasing powers of $y$ to obtain

$$
\begin{aligned}
(1-z) R_{s}(z) & =z(1-y z) \sum_{n \geq 0} \prod_{k=1}^{n} y\left(z+z^{2^{k}}\right) \\
& =z \sum_{n \geq 0} \prod_{k=1}^{n} y\left(z+z^{2^{k}}\right)-z^{2} y \sum_{n \geq 0} \prod_{k=1}^{n} y\left(z+z^{2^{k}}\right) \\
& =z \sum_{n \geq 0} y^{n} \prod_{k=1}^{n}\left(z+z^{2^{k}}\right)-z^{2} y \sum_{n \geq 1} \prod_{k=1}^{n-1} y\left(z+z^{2^{k}}\right) \\
& =z \sum_{n \geq 0} y^{n} \prod_{k=1}^{n}\left(z+z^{2^{k}}\right)-z^{2} \sum_{n \geq 1} y^{n} \prod_{k=1}^{n-1}\left(z+z^{2^{k}}\right) \\
& =z+z \sum_{n \geq 1} y^{n}\left(z+z^{2^{n}}\right) \prod_{k=1}^{n-1}\left(z+z^{2^{k}}\right)-z^{2} \sum_{n \geq 1} y^{n} \prod_{k=1}^{n-1}\left(z+z^{2^{k}}\right) \\
& \left.=z+z \sum_{n \geq 1} y^{n}\left(z+z^{2^{n}}\right) \prod_{k=1}^{n-1}\left(z+z^{2^{k}}\right)-z \prod_{k=1}^{n-1}\left(z+z^{2^{k}}\right)\right) \\
& =z+z \sum_{n \geq 1} y^{n} z^{2^{n}} \prod_{k=1}^{n-1}\left(z+z^{2^{k}}\right) .
\end{aligned}
$$

Note that this last expression is equal to $\mathcal{D}_{s}(z)$ by $(7)$. 